\title{
Evaluation of the Quality of Life and the Frequency of Attention Deficit Hyper Activity Disorder in Children with Epilepsy
}

Mojtaba Fazel ${ }^{1}$, Mostafa Almasi Dooghaee ${ }^{2 *}$, Faeze Ghasemi ${ }^{2}$, Paria Hebrani ${ }^{3}$, Farah Ashrafzadeh

${ }^{1}$ Department of Emergency Medicine, Imam-Reza Hospital, Mashhad University of Medical Sciences, Mashhad, Iran ${ }^{2}$ Department of Neurology, Rasool-Akram Hospital, Tehran, Iran

${ }^{3}$ Department of Psychiatry, Ebn Sina Hospital, Mashhad University of Medical Sciences, Mashhad, Iran

${ }^{4}$ Department of Pediatrics Neurology, Ghaem Hospital, Mashhad University of Medical Sciences, Mashhad, Iran

\section{A BSTRACT}

Introduction: Epilepsy causes significant effects on child's physical and mental functions and is associated with different psychiatric disorders. The aim of this study was to compare the frequency of attention deficit hyperactivity disorder (ADHD) and quality of life (QOL) in children with epilepsy. Materials and Methods: This study was conducted on 90 children that divided to 3 groups: chronic epilepsy, recent epilepsy (with diagnosis of less than a month), and control groups. In the first stage, all patients were screened with a questionnaire containing demographic data, ADHD Rating Scale (ADHD-RS), Child-self report and Parent-proxy report as well as Pediatric Quality of Life Inventory TM (Peds QL ${ }^{\mathrm{TM}}$ ) forms. Then, the children were evaluated for definite diagnosis of ADHD according to DSM-IV criteria. Results: ADHD was diagnosed in $43.3 \%, 36.7 \%$ and $6.7 \%$ of chronic epilepsy, recent epilepsy, and control groups, respectively. There was a significant correlation between Parent-proxy report QOL score and the type of epilepsy. In analysis of QOL subgroups, the score of physical function and school performance were different in three groups. Children with ADHD had significantly lower total QOL score than non-ADHD children. Conclusion: The physical function, school performance, and total QOL score of chronic epileptic children are lower than recent epileptic and non-epileptic children. The ADHD is more common in epileptic children than normal children. Screening and early treatment of ADHD in epileptic children may improve their QOL.

\section{Key words:}

1. Epilepsy

2. Attention Deficit Disorder with Hyperactivity

3. Quality of Life

4. Child

* Corresponding Author: Mostafa Almasi Dooghaee

E-mail:a_mostafa108@yahoo.com 


\section{بررسى كيفيت زندگى و فراوانى اختلال كمبود توجه -بيش فعالى دركودكان مبتلا به صرع}

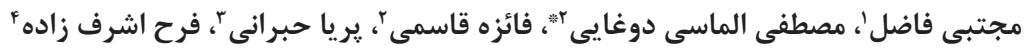

كَروه طب اوررانس، بيمارستان امام رضا، دانشعاه علوم يزشكى مشهد، مشهد، ايران

rكروه نورولوزى، بيمارستان رسول اكرم، تهران، ايران

"كروه روانيزشكى، بيمارستان ابن سينا، دانشكاه علوم يزشكى مشهد، مشهد، ايران

×َّروه مغز و اعصاب كودكان، بيمارستان قائم، دانشكاه علوم يزشكى مشهد، مشهد، ايران

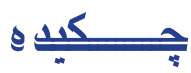

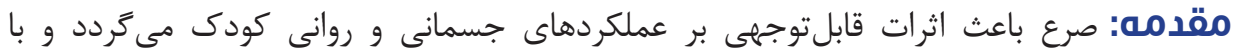

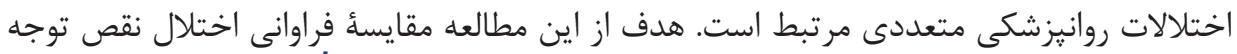

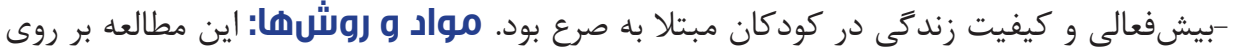

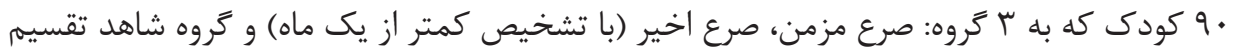

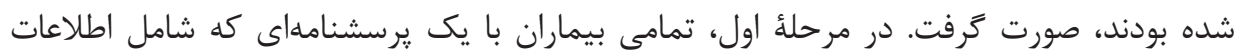

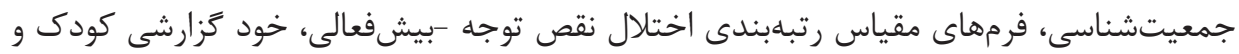

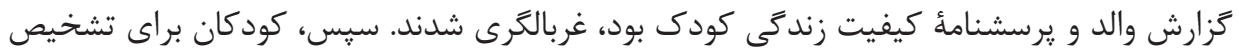

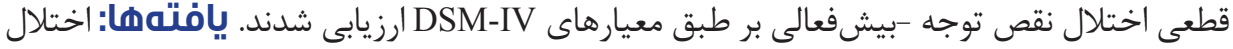

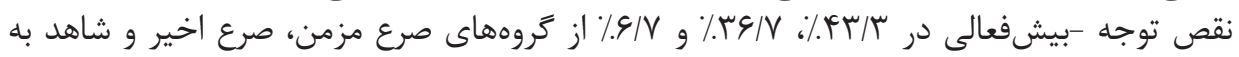

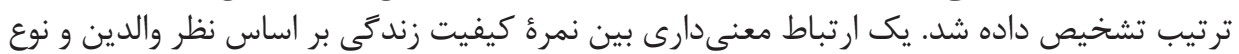

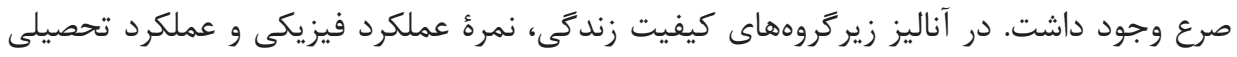

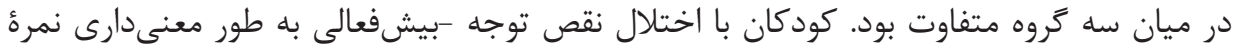

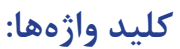

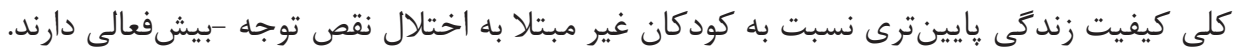

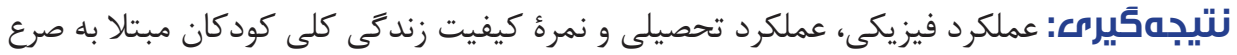
r. r. اختلال نقص توجه -بيشفعالى زئل

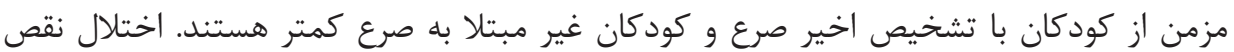

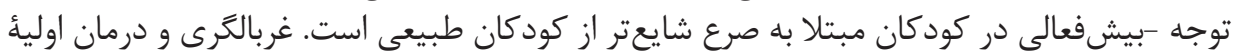

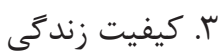

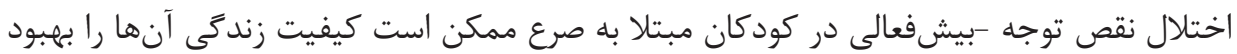


آنها كذشته بود و تحت درمان با داروهاى ضد صرع بودند و و

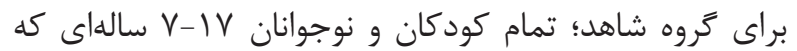

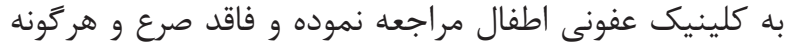

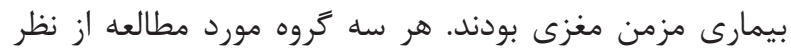

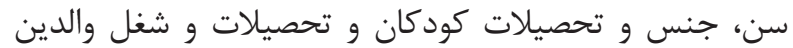

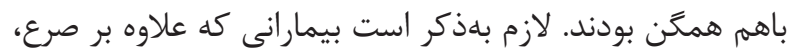

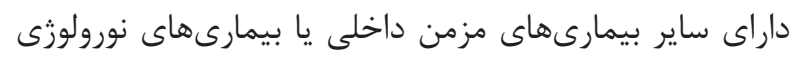

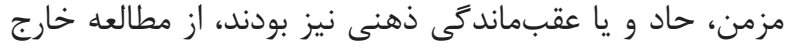

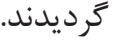

جمعآورى اطلاعات در دو مرحله انجام شد. مرحلة اول أنداد

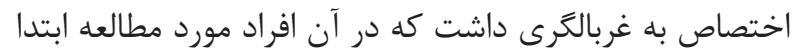

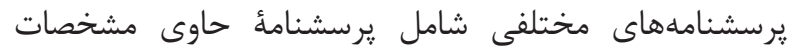

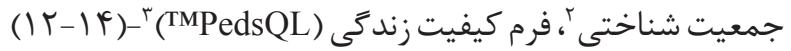

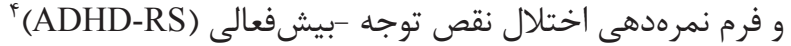

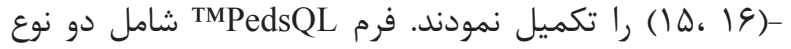

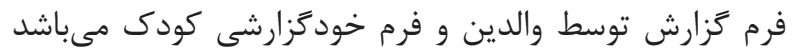

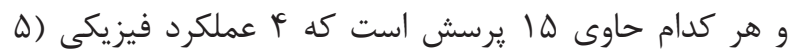

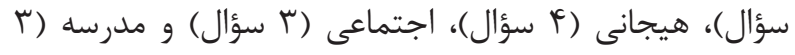

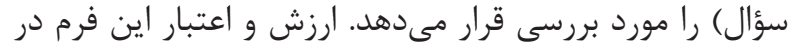

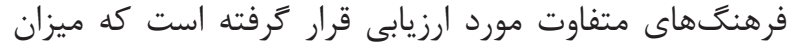

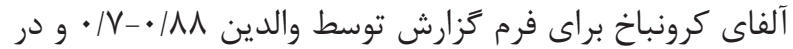

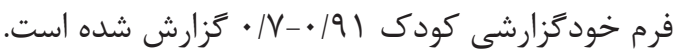

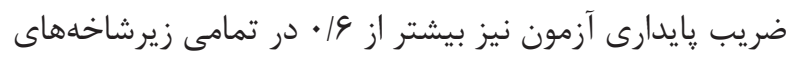

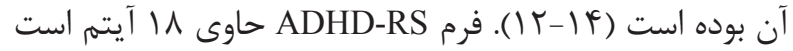

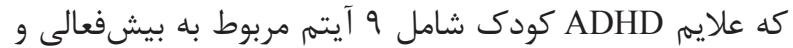

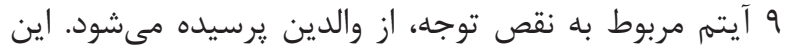

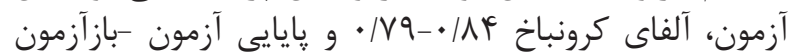

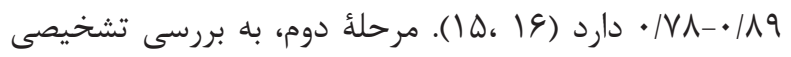

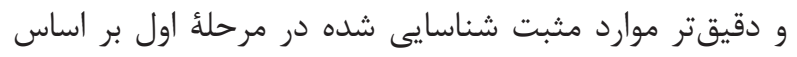

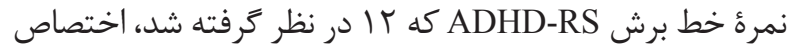

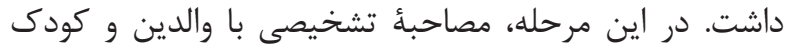

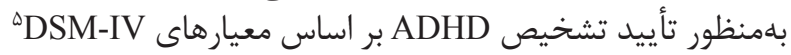

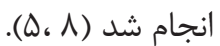

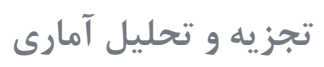

اطلاعات به دست آمده كدَذارى شده و با استفاده از نرمافزار

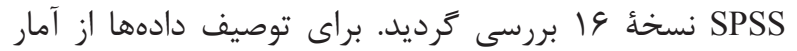

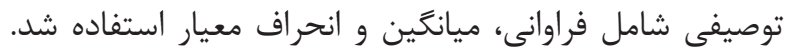

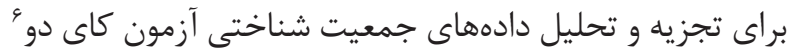

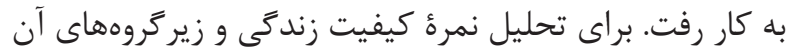

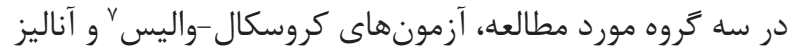

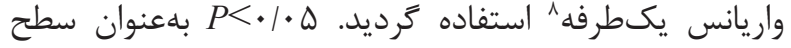
معنى دارى در نظر كرفته شد.

${ }^{1}$ Attention deficit hyperactivity disorder

${ }^{2}$ Demographic

${ }^{3}$ Pediatric quality of life inventory TM

${ }^{4}$ Attention deficit hyperactivity disorder rating scale
مقدمه

صرع در بين بيمارىهاى جسمانى دوران كودكى، از شايعترين

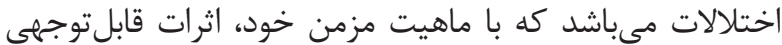

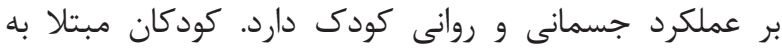

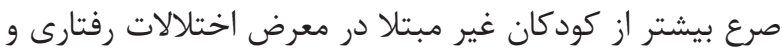

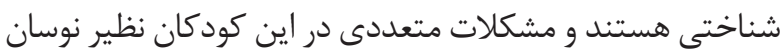

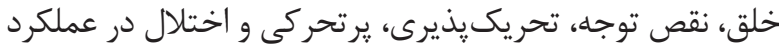

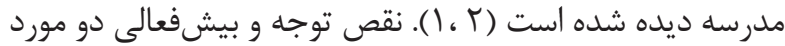

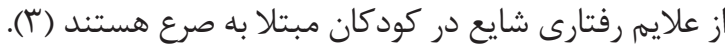

اختلال نقص توجه -بيشفعالى (ADHD)' بهعنوان يكى از

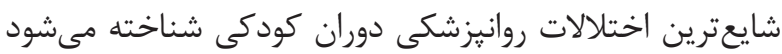

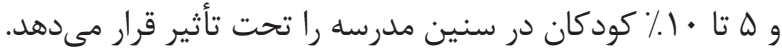

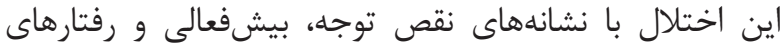

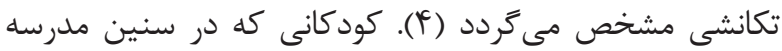

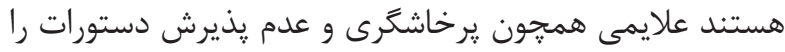

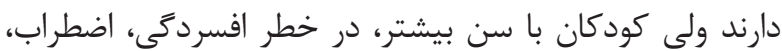

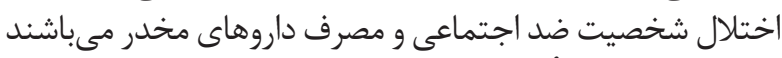

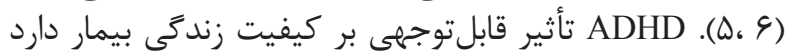

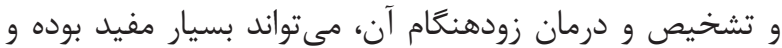

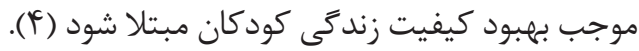

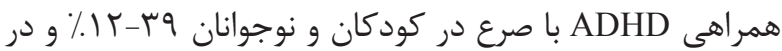

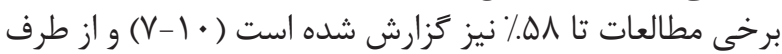

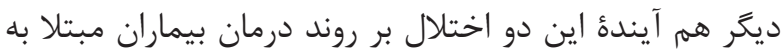

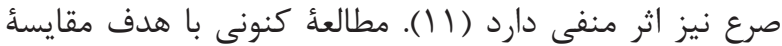

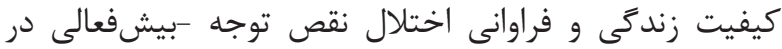

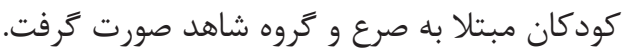

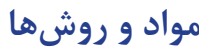

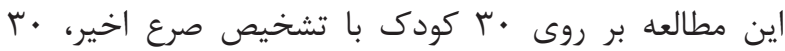

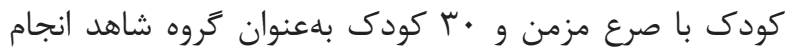

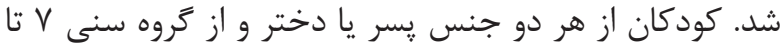

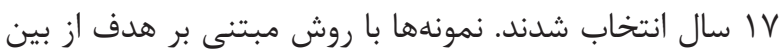

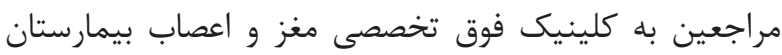

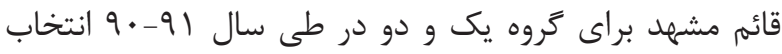

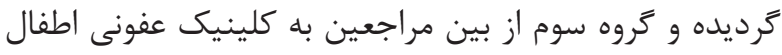

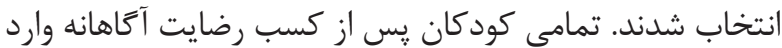
مطالعه كرديدند. معيارهاى ورود به مطالعه بدين گَونه بود: براى گرووه صرع اخير؛

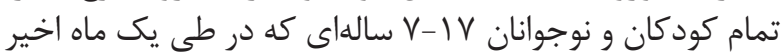

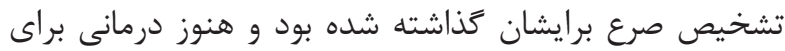

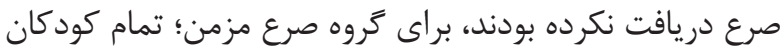

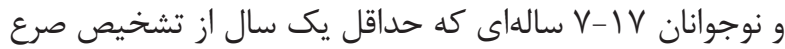

\footnotetext{
${ }^{5}$ Diagnostic and statistical manual of mental disorders, $4^{\text {th }}$ edition ${ }^{6}$ Chi square

${ }^{7}$ Kruskal-Wallis

${ }^{8}$ One-way ANOVA test
} 


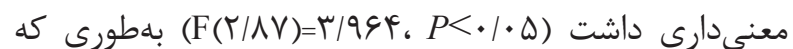
نمرة كلى كيفيت زند

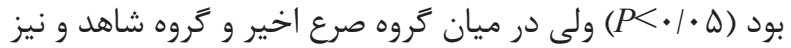

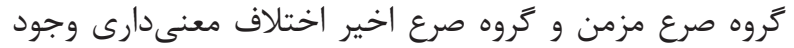

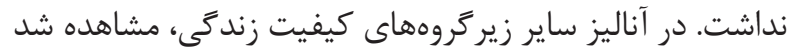

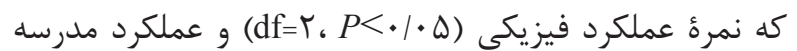

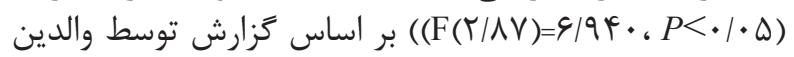

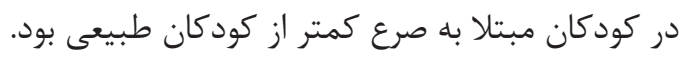
بحث و نتيجه كيرى

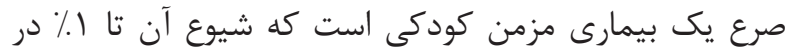

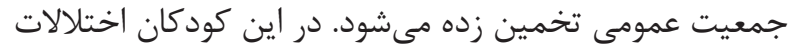

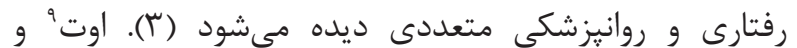

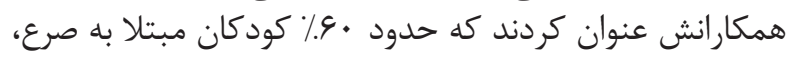

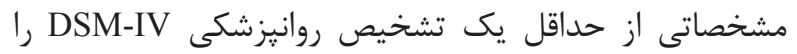

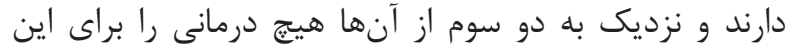
اختلالات دريافت نمى كنند (IV) إين مسئله تأثير بسزائى دائ در اين

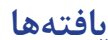

ميانكَين سنى كودكان مورد مطالعه

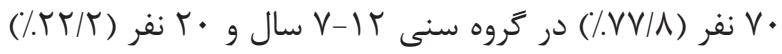

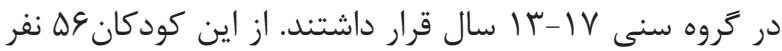
( )

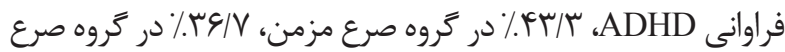

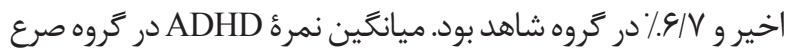

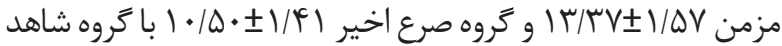

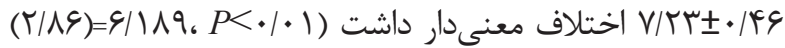

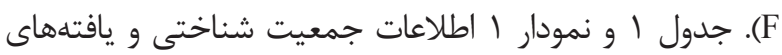
رادر سه كروه مورد مطالعه نشان مى النهند.

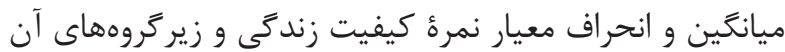

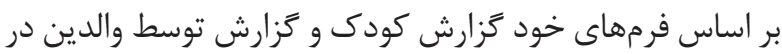

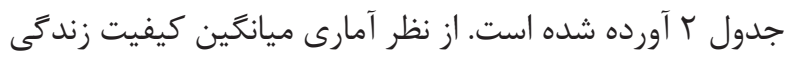

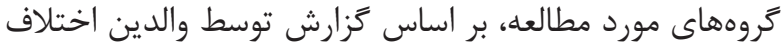

جدول ا- مشخصات جمعيت شناختى و فراوانى ADHD دركودكان مبتلا به صرع و شاهد.

\begin{tabular}{|c|c|c|c|c|c|}
\hline كل ل & كروه شاهد & كروه صرع اخير & كروه صرع مزمن & & \\
\hline$\% \vee \vee / \Lambda$ & $\%$ VT/r & $\%$ & $\% \vee \& / V$ & rال V- سال & \\
\hline \% & $\%$ r re/V & $\% 1 \% / V$ & \% & Vا-rاسال & \\
\hline $1.9 \mathrm{~T} / \mathrm{T}$ & $\% .9$. & $\% .9$. & $\% .94 / \mathrm{V}$ & ل يسر & \\
\hline$\% \mathrm{rV} / \mathrm{A}$ & $\%$. & $\%$. & \% & دختر & \\
\hline$\% \notin \Delta / \varepsilon$ & $\% F r / r$ & $\% r r / r$ & $\% .9$ & دارد & \\
\hline$\% \Delta F / F$ & $\% \Delta \varphi / V$ & $1.99 / \mathrm{V}$ & $\%$. & ندارد & 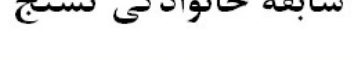 \\
\hline$\% / r \wedge / 9$ & $\% / \mathrm{V}$ & $\%$ rs/V & $\%$ Fr/r & & فراوانى ADHD \\
\hline
\end{tabular}

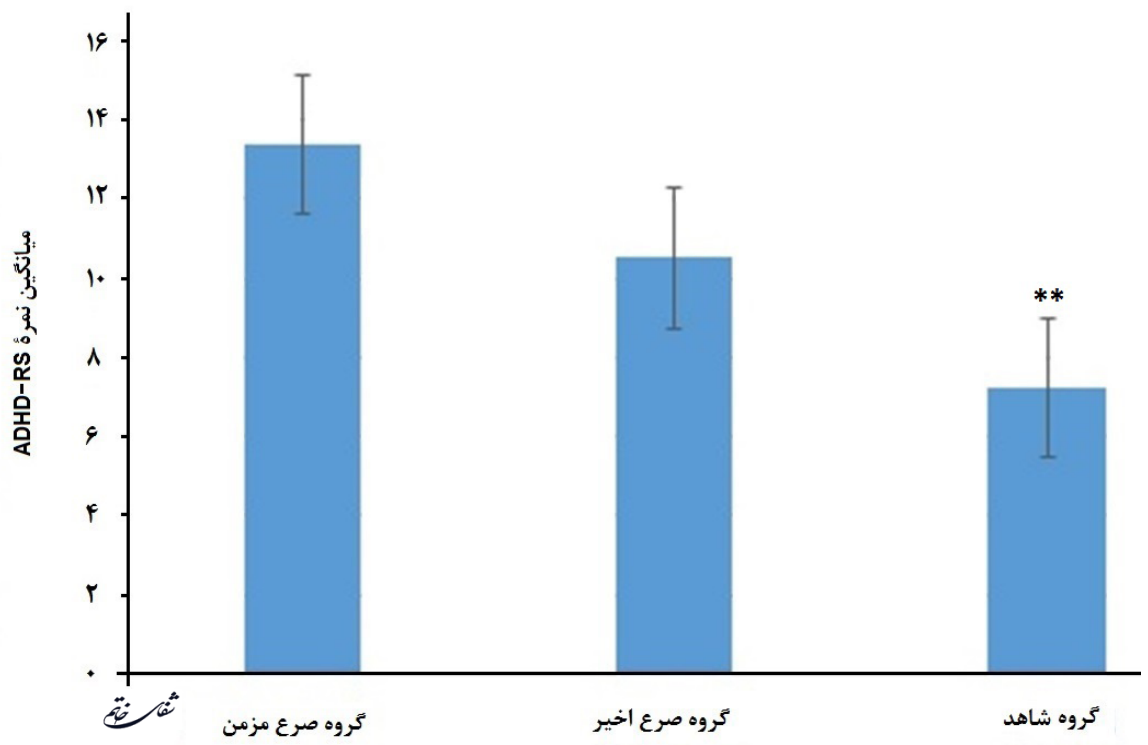

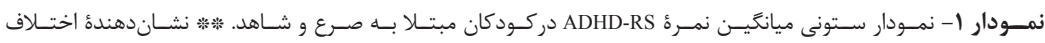


جدول r- ميانكَين و انحراف معيار هر يك از زيركروهاى كيفيت زندكى خودَّارش كودى يا كزارش توسط والدين، دركودكان مبتلا به صرع و شاهد.

\begin{tabular}{|c|c|c|c|c|c|c|c|c|c|c|}
\hline \multicolumn{2}{|c|}{ نمرة كلى كيفيت زندلى } & \multicolumn{2}{|c|}{ عملكرد مدرسه } & \multicolumn{2}{|c|}{ مملكرد اجتماعى } & \multicolumn{2}{|c|}{ عملكرد هيجاتى } & \multicolumn{2}{|c|}{ عملكرد فيزيكى } & \\
\hline تزارش توسط & خوقئارش كورى & كزارش توسط & خوقثزارش كودى & تزارش توسط & خودكزارش كودى & كزارش توسط & خونّزارش كودى & تزارش توسط & خوقئزارش كودى & \\
\hline$A 1 / V \cdot \pm r / r$ & $\Delta F / \Delta Y \pm 1 / q$ & $v \xi|\cdot \pm \notin /|$ & $\Delta r / \| r \pm r / r$ & $\Lambda \cdot / r \varphi \pm r / \Lambda$ & $\Delta T / a r \pm r / T$ & $V \Lambda / \Delta \Lambda \pm r / A$ & $\Lambda 1 / 9 \Delta \pm r / 1$ & $q r / A r \pm r / r$ & $q r / 9 v \pm r / r$ & صرع مزمن \\
\hline$\Delta F / \Delta r \pm r / 1$ & $\Lambda q / V \cdot \pm 1 / \Delta$ & $\Delta F / \Delta r \pm r / V$ & $\Lambda g / 4 \cdot \pm r / q$ & $A 1 / A 9 \pm r / r$ & $\Lambda \mathrm{Q} / \cdot V \pm r / q$ & $V N / \Delta F \pm r / F$ & $A r / \Delta F \pm r / A$ & $q \Gamma / \Delta \cdot \pm \Gamma /$. & $q r / q V \pm r / f$ & ص ع ع اخير \\
\hline$\Lambda 9 / \Delta \cdot \pm 1 / \cdot$ & $\Lambda q / F \cdot \pm 1 / 1$ & $q r / \Lambda q \pm \mid / V$ & $q \pi / T Y \pm 1 / 9$ & $9 \cdot / / r \pm r / r$ & $\Lambda 9 / r r \pm r / r$ & $\Lambda \mathrm{Q} / 9 \mathrm{G} \pm 1 / \mathrm{Q}$ & $\Lambda d / F| \pm| / r$ & $\wedge 9 / \cdot * \pm 1 / 9$ & $q \cdot / \Lambda r \pm 1 / p$ & كروه شاهد \\
\hline$+1+4 r$ &.$/ 1 \cdot 9$ &.$\cdots p$ & .1 .49 & $. / 1+4$ & . & . & $.1 \% v 9$ & $.1 \%$ & 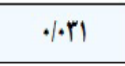 & P-value \\
\hline
\end{tabular}

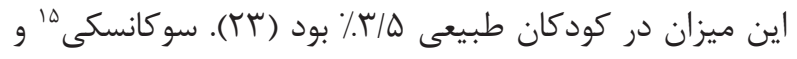

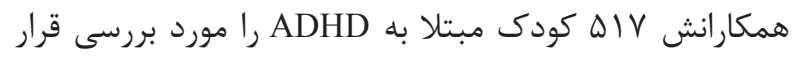

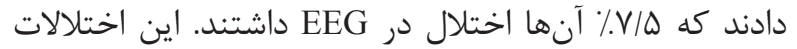

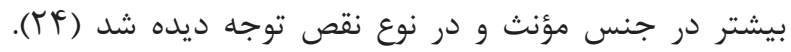

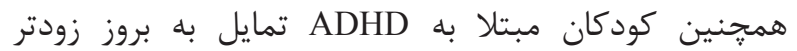

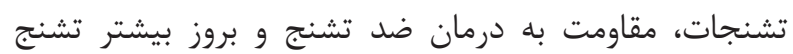
استاتوس 19 دارند (Y) مقاوت (4).

بالاتر بودن شيوع ADHD در كودكان مبتلا به صرع در مطالعئ

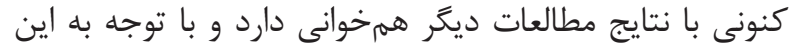

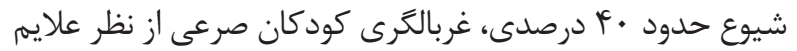
ADHD

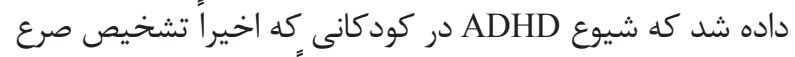

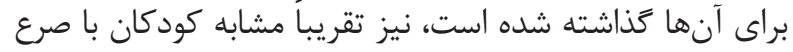

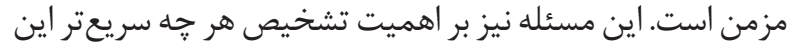
اختلال روانيزشكى در كودكان صرعى، صحه مى مذارد. در مورد اينكه آيا اين همراهى ميان صرع و ADHD به صورت

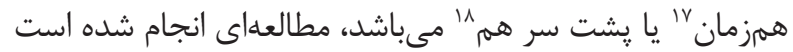

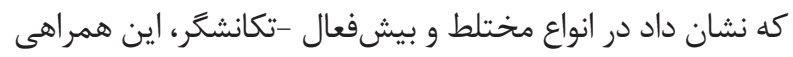

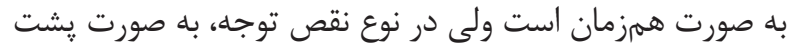

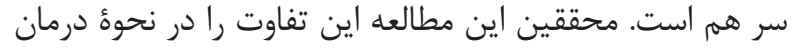

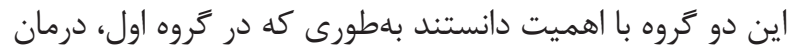

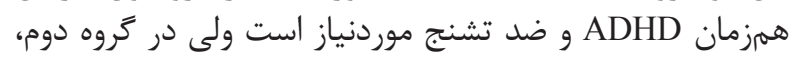

تأكيد بيشتر بر روى درمان ضد تشنج بايد باشد (ه) (Y). در مطالعة ما مشخص شد كه ميانگين نمره كيفيت زندگى

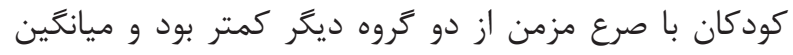

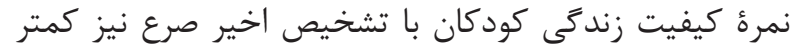

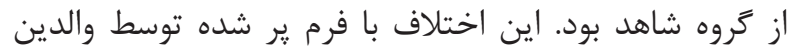

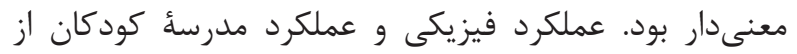
ديد

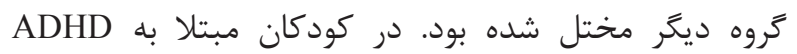

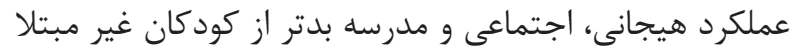

\footnotetext{
${ }^{10}$ Lennox-Gastaut syndrome

${ }^{11}$ Generalized tonic-clonic

${ }^{12} \mathrm{McDermott}$

${ }^{13}$ Davis

${ }^{14}$ Electroencephalography
}

كيفيت زندكى اين كودكان دارد و نياز به ارزيابىهاى تشخيصى

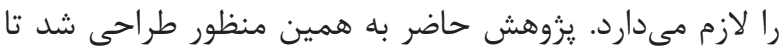

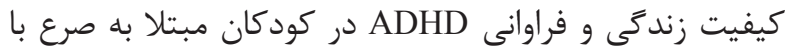
كودكان غير مبتلا مقايسه زَرد

همجنين در اين يزوهش، تأثير ازمان صرع بر بروز ADHD

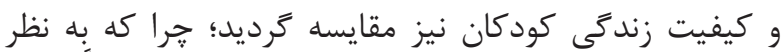

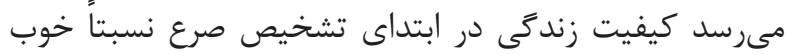
است و با ازمان صرع افت مئيت كند (1) (1).

در اين يزوهش، شيوع ADHD در گروه شاهد مورد بررسى

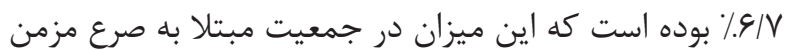

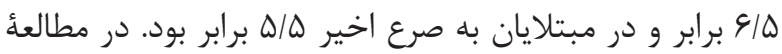

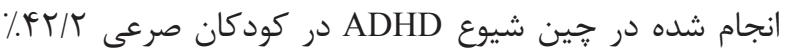

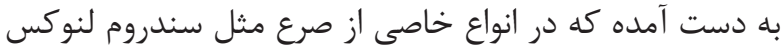

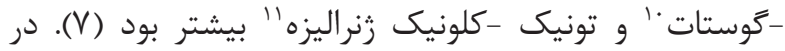

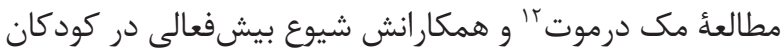

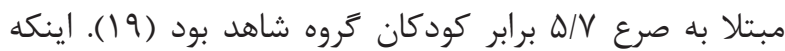

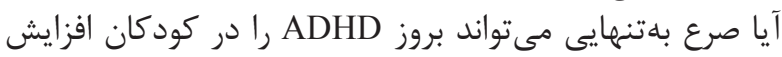
دهد و يا ساير عوامل همراه با صرع منجر به به اين افزايش برون بروز

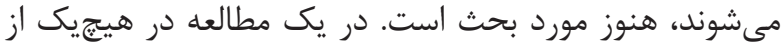

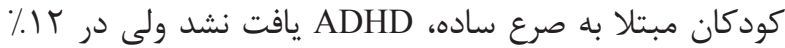

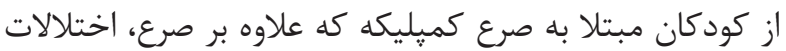

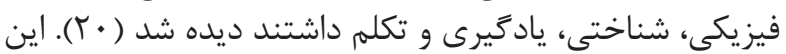

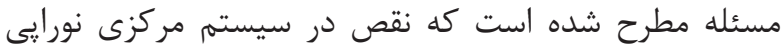
نفرين مىتواند عامل بيشتر بودن نوع نقص توجه دره در كودكان

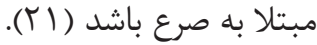
از طرف ديخر در مبتلايان به ADHD نيز بروز صرع بالاتر است.

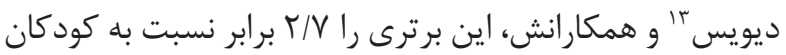

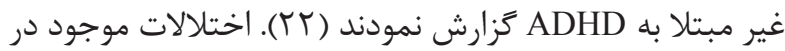

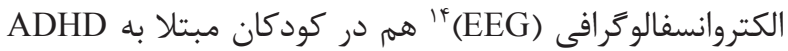

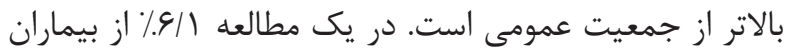
مبتلا به ADHD تغييرات تشنجى در

\footnotetext{
${ }^{15}$ Socanski

${ }^{16}$ Status epilepticus

${ }^{17}$ Concurrent

${ }^{18}$ Successive
} 
بهترى را در كودكان مبتلا به ADHD نسبت به به والدين خود،

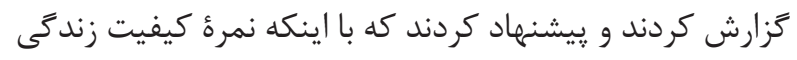

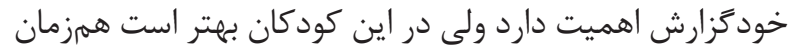
از نظرات والدين هم استفاده شود (•(ب). در مجموع با نتايج اين مطالعه مىتوان كفت كه كيفيت زندكى

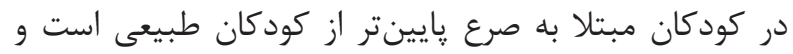

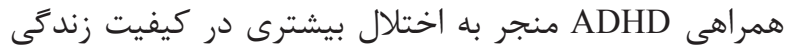

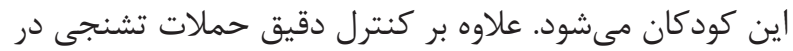

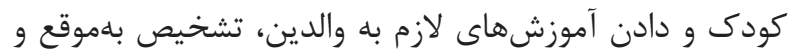

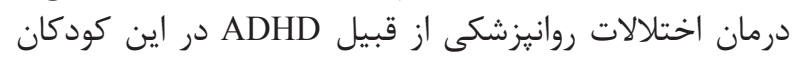
كمك بسزايى در بهبود كيفيت زندى اخلى آنها خواهد ندان نمود.

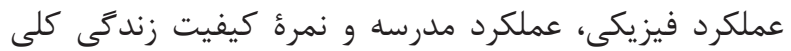

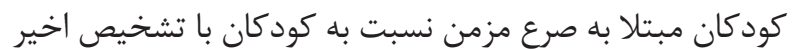

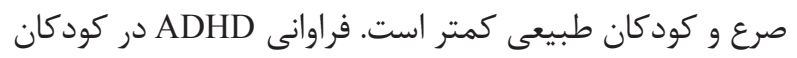

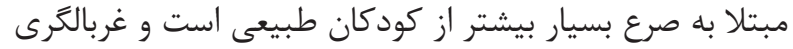

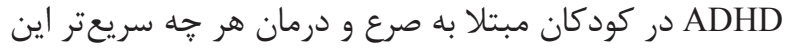

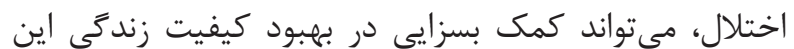

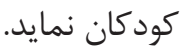

نقاط قوت اين مطالعه، داشتن كروه شاهد و و مقايسأ كيفيت

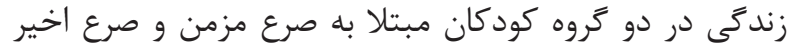

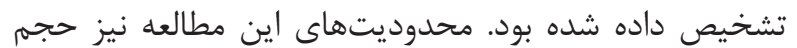

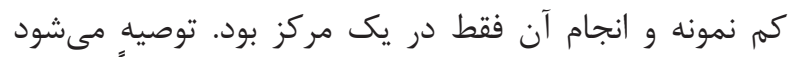

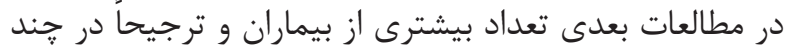

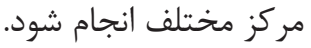

1. Fenichle GM. Paroxysmal disorders. Clinical pediatric neurology: A signs and symptoms approach: expert consult-online and print. $6^{\text {th }}$ ed. Philadelphia Saunders Elsevier. 2009; p. 10-23.

2. Camfield PR, Camfield CS. Pediatric epilepsy in: Swaiman's pediatric neurology 5th ed. Philadelphia Saunders Elsevier. 2012; p. 800-91.

3. Dunn DW, Austin JK. Differential diagnosis and treatment of psychiatric disorders in children and adolescents with epilepsy. Epilepsy Behav. 2004; 5 Suppl 3: S10-7.

4. Reilly CJ. Attention deficit hyperactivity disorder (ADHD) in childhood epilepsy. Res Dev Disabil. 2011; 32(3): 883-93.

\footnotetext{
${ }^{19}$ Dafoulis

${ }^{20}$ Kalyva

${ }^{21}$ Speechley
}

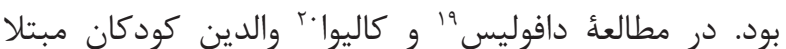

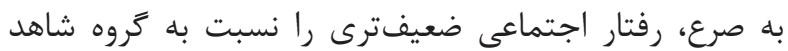

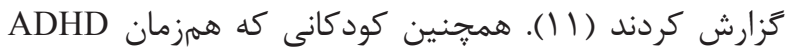

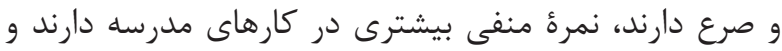

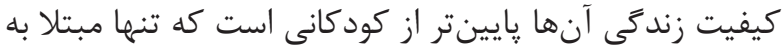

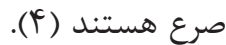

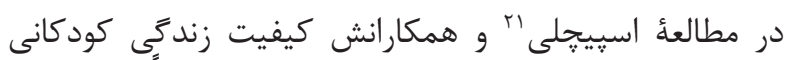

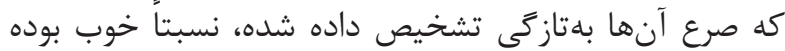

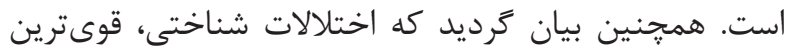

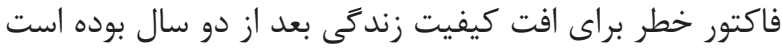

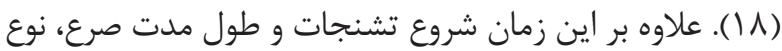

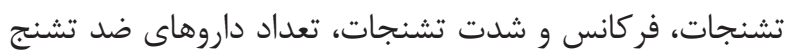

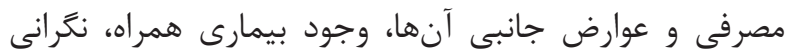

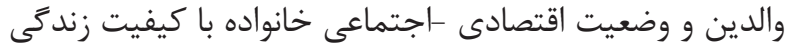
در كودكان مبتلا به صرع مرتبط هستند (؟) (؟).

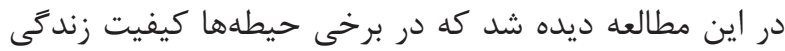

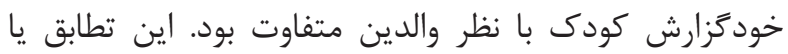

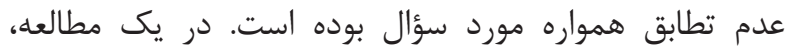

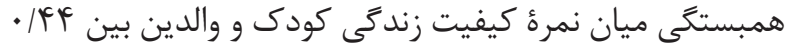

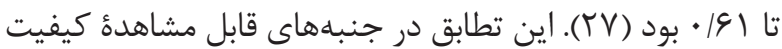

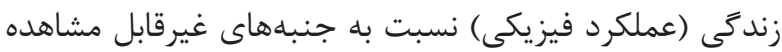

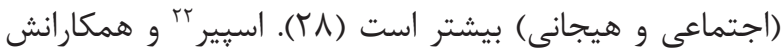

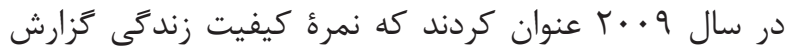

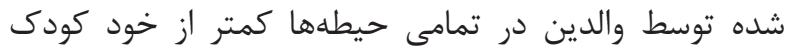

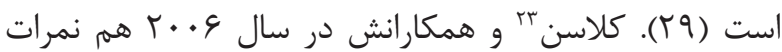

\section{منابع}

5. Fischer M, Barkley RA, Smallish L, Fletcher K. Young adult follow-up of hyperactive children: selfreported psychiatric disorders, comorbidity, and the role of childhood conduct problems and teen CD. J Abnorm Child Psychol. 2002; 30(5): 463-75.

6. Young S, Amarasinghe JM. Practitioner review: Nonpharmacological treatments for ADHD: A lifespan approach. J Child Psychol Psychiatry. 2010; 51(2): 116-33.

7. Han Y, Qin J, Jiang YW, Chen J, Ji XN, Lin Q. Comorbidity of attention deficit hyperactivity disorder in children with epilepsy. Zhongguo Dang Dai Er Ke Za Zhi. 2012; 14(2): 89-92.

8. Dunn DW, Kronenberger WG. Childhood epilepsy, attention problems, and ADHD: review and practical
${ }^{22}$ Speyer
${ }^{23}$ Klassen 
considerations. Semin Pediatr Neurol. 2005; 12(4): 222-8.

9. Loutfi KS, Carvalho AM, Lamounier JA, Nascimento Jde A. ADHD and epilepsy: contributions from the use of behavioral rating scales to investigate psychiatric comorbidities. Epilepsy Behav. 2011; 20(3): 484-9.

10. Cornelio-Nieto JO, Borbolla-Sala ME, GallegosDimas A. Electroencephalographic alterations in children with attention deficit hyperactivity disorder. Rev Neurol. 2011; 52 Suppl 1: S97-101.

11. Dafoulis V, Kalyva E. Factors associated with behavioral problems in children with idiopathic epilepsy. Epilepsy Res. 2012; 100(1-2): 104-12.

12. Chen X, Origasa H, Ichida F, Kamibeppu K, Varni JW. Reliability and validity of the pediatric quality of life inventory (PedsQL) short form 15 generic core scales in Japan. Qual Life Res. 2007; 16(7): 1239-49.

13. Gkoltsiou K, Dimitrakaki C, Tzavara C, Papaevangelou V, Varni JW, Tountas Y. Measuring health-related quality of life in Greek children: psychometric properties of the Greek version of the pediatric quality of life inventory(TM) 4.0 generic core scales. Qual Life Res. 2008; 17(2): 299-305.

14. Laaksonen C, Aromaa M, Heinonen OJ, Suominen S, Salanterä S. Paediatric health-related quality of life instrument for primary school children: cross-cultural validation. J Adv Nurs. 2007; 59(5): 542-50.

15. Zhang S, Faries DE, Vowles M, Michelson D. ADHD rating scale IV: Psychometric properties from a multinational study as a clinician-administered instrument. Int J Methods Psychiatr Res. 2005; 14(4): 186-201.

16. Döpfner M, Steinhausen HC, Coghill D, Dalsgaard S, Poole L, Ralston SJ, et al. Cross-cultural reliability and validity of ADHD assessed by the ADHD rating scale in a pan-European study. Eur Child Adolesc Psychiatry. 2006; 15 Suppl 1: I46-55.

17. Ott D, Siddarth P, Gurbani S, Koh S, Tournay A, Shields WD, et al. Behavioral disorders in pediatric epilepsy: unmet psychiatric need. Epilepsia. 2003; 44(4): 591-7.

18. Speechley KN, Ferro MA, Camfield CS, Huang W, Levin SD, Smith ML, et al. Quality of life in children with new-onset epilepsy: a 2-year prospective cohort study. Neurology. 2012; 79(15): 1548-55.

19. McDermott S, Coker AL, Mani S, Krishnaswami S, Nagle RJ, Barnett-Queen LL, et al. Population-based analysis of behavior problems in children with cerebral palsy. J Pediatr Psychol. 1996; 21(3): 447-63.

20. Davies S, Heyman I, Goodman R. A population survey of mental health problems in children with epilepsy. Dev Med Child Neurol. 2003; 45(5): 292-5.

21. Hesdorffer DC, Ludvigsson P, Olafsson E, Gudmundsson G, Kjartansson O, Hauser WA. ADHD as a risk factor for incident unprovoked seizures and epilepsy in children. Arch Gen Psychiatry. 2004; 61(7): 731-6.

22. Davis SM, Katusic SK, Barbaresi WJ, Killian J, Weaver AL, Ottman R, et al. Epilepsy in children with attention-deficit/hyperactivity disorder. Pediatr Neurol. 2010; 42(5): 325-30.

23. Richer LP, Shevell MI, Rosenblatt BR. Epileptiform abnormalities in children with attention deficit/ hyperactivity disorder. Pediatr Neurol. 2002; 26(2): 125-9.

24. Socanski D, Herigstad A, Thomsen PH, Dag A, Larsen TK. Epileptiform abnormalities in children diagnosed with attention deficit/hyperactivity disorder. Epilepsy Behav. 2010; 19(3): 483-6.

25. Noeker M, Haverkamp F. Neuropsychological deficiencies as a mediator between CNS dysfunction and inattentive behaviour in childhood epilepsy. Dev Med Child Neurol. 2003; 45(10): 717-8.

26. Ferro MA. Risk factors for health-related quality of life in children with epilepsy: a meta-analysis. Epilepsia. 2014; 55(11): 1722-31.

27. Theunissen NC, Vogels TG, Koopman HM, Verrips GH, Zwinderman KA, Verloove-Vanhorick SP, et al. The proxy problem: child report versus parent report in health-related quality of life research. Qual Life Res. 1998; 7(5): 387-97.

28. Eiser C, Morse R. Can parents rate their child's health-related quality of life? Results of a systematic review. Qual Life Res. 2001; 10(4): 347-57.

29. Speyer E, Herbinet A, Vuillemin A, Chastagner P, 
Briançon S. Agreement between children with cancer and their parents in reporting the child's health-related quality of life during a stay at the hospital and at home. Child Care Health Dev. 2009; 35(4): 489-95.
30. Klassen AF, Miller A, Fine S. Agreement between parent and child report of quality of life in children with attention-deficit/hyperactivity disorder. Child Care Health Dev. 2006; 32(4): 397-406. 